\title{
Statistical analysis of the long-term visual light curve parameters of dwarf novae
}

\author{
T. Ak ${ }^{1}$, M. T. Ozkan² , and J. A. Mattei ${ }^{3}$ \\ 1 University of Istanbul, Faculty of Science, Department of Astronomy and Space Sciences, \\ 34452 University-Istanbul, Turkey \\ 2 Istanbul University Observatory Research and Application Center, 34452 University-Istanbul, Turkey \\ 3 American Association of Variable Star Observers, 25 Birch Street, Cambridge, MA 02138, USA
}

Received 15 February 2002 / Accepted 9 April 2002

\begin{abstract}
The long-term visual light curve parameters of 36 dwarf novae are measured. The data is anayzed in search of likely relationships among the light curve parameters and system parameters. New relations of the orbital period with the mean light curve parameters are given. Our findings for the correlation analysis of individual systems agree with some of the results in previous studies. Almost all of the systems in this study have a bimodal frequency distribution of the outburst duration. By comparing the observational correlations with their theoretical counterparts, we find that the value of the viscosity parameter of the disk instability model is $\alpha_{\text {hot }} \approx 0.2$.
\end{abstract}

Key words. stars: dwarf novae - stars: novae, cataclysmic variables - stars: variables: general

\section{Introduction}

Cataclysmic variables are short period binary stars consisting of a late-type, near-main-sequence star (the secondary) filling its Roche lobe and transferring matter onto a white dwarf (the primary). Dwarf novae are a subclass of cataclysmic variables displaying quasi-periodic outbursts of 2-6 magnitudes that repeat every $10-500$ or more days. Dwarf novae can be divided into three main subtypes (Mattei 1990). U Geminorum (U Gem) stars exhibit only normal outbursts. Z Camelopardalis (Z Cam) stars show evidence of standstills. SU Ursae Majoris (SU UMa) stars possess superoutbursts. Quasi-periodic outburst behaviour of dwarf novae is more easily explained by viscous processes acting in the accretion disk (Smak 1984; Cannizzo et al. 1988; Cannizzo 1993; Smak 1998; Hameury et al. 1998).

Visual photometric data of dwarf novae have been collected for decades, by some associations such as the AAVSO and RASNZ. The long-term visual observations represent a wealth of information about statistical correlations of the long-term light curve parameters that can give some clues for outburst models and accretion disk studies. We have attempted to use the long-term light curves of dwarf novae to derive statistical correlations of

Send offprint requests to: T. Ak, e-mail: tanselak@istanbul.edu.tr the long-term light curve parameters. Previous efforts in this regard have been made for some individual systems (e.g., Sterne \& Campbell 1934; Smak 1985; Cannizzo \& Mattei 1992; Mohanty \& Schlegel 1995). Results using compilations from several sources have been reported by Bailey (1975), Jacchia (1975), van Paradijs (1983), Gieger (1987), Antipova (1987), Richter \& Brauer (1989) and Vogt (1995).

Previous studies suffer from a lack of consistency in measurement as they represent compilations, and often are based on data consisting of a few outbursts. To counteract this deficiency, Szkody \& Mattei (1984, hereafter referred to as SM84) have measured the light curve parameters and found statistical correlations of the parameters for 21 dwarf novae. However, SM84 is based on data covering 1000 days of observations.

In order to extend the analysis of the long-term light curve parameters to a larger sample of dwarf novae, we have analyzed all the available light curves obtained by the AAVSO and RASNZ on 36 systems.

\section{Data and analysis}

Table 1 presents the 36 systems studied and general characteristics of data. Because we use only well-observed outbursts, the number of outbursts is small for those systems lacking sufficient number of observations. The light curve 


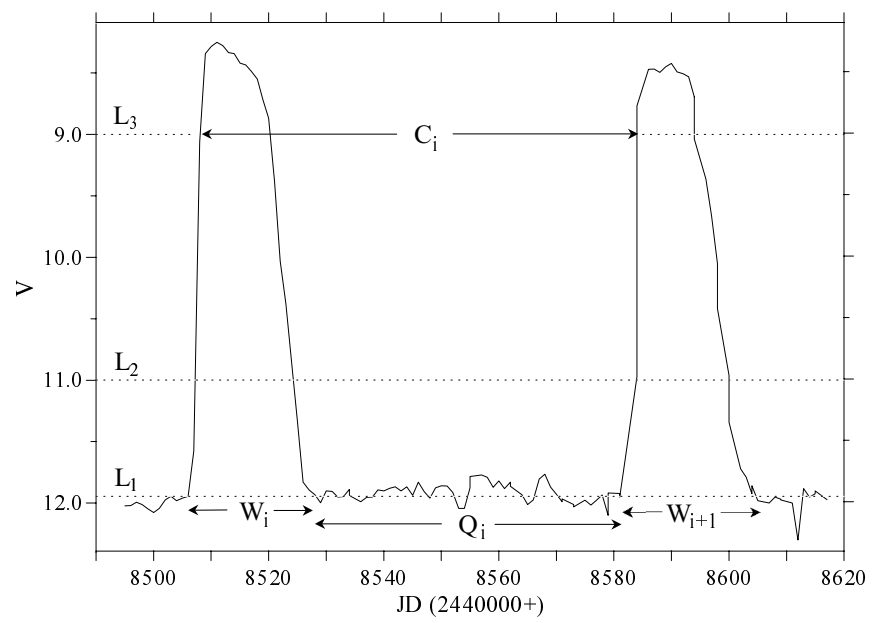

Fig. 1. Illustrative definitions of the outburst duration $W$, quiescent interval $Q$ and cycle time $C$. The subscripts " $i-1$ " and " $i$ " denote the parameters for intervals preceeding and following the "ith" outburst, respectively. $L_{1}, L_{2}$ and $L_{3}$ correspond to the first, second and third reference levels (dotted lines), respectively.

parameters measured in this study are the outburst duration, quiescent interval, cycle time, decline rate and rise rate.

Definitions of the outburst duration, quiescent interval and the cycle time are illustrated in Fig. 1 for an outburst of SS Cyg. The light curve parameters were measured at three reference levels by the aid of a computer program: 1) The first reference level is the quiescent magnitude of each outburst. Because the quiescent magnitude is variable on long time scales, this reference level does not correspond to a constant brightness. We choose to measure the outburst duration $W$ as the interval from leaving quiescence to return to quiescence. The quiescent interval $Q$ is the time spent at the quiescent level following the outburst. Although this reference level introduces some difficulties for systems with faint quiescent levels, we should emphasize that the measures at the quiescent level would be a better representation for general comparison to outburst models. 2) For a given system, the second reference level is a constant brightness above the average quiescent magnitude. 3) Similarly, the third reference level is a constant brightness below the average maximum magnitude. The second and third reference levels are listed in Table 2 for each system. We define the cycle time $C$ at the third reference level as the interval from the start of an outburst to the start of the next, by assuming the third reference level to be the base of outbursts. The decline and rise rates are calculated between the second and third reference levels. The rise rate, $\tau_{\mathrm{r}}$, is the time spent to increase a systems's brightness one magnitude on the rising branch of an outburst. Similarly, the decline rate, $\tau_{\mathrm{d}}$, is the time spent to decrease a systems's brightness one magnitude on the declining branch of an outburst.

Table 2 includes the mean light curve parameters. Ten of the systems in SM84 overlap with our sample. For these
Table 1. The names, orbital periods ( $P_{\text {orb }}$, in hours), subtypes, time intervals of observations, number of observations $\left(N_{\text {obs }}\right)$ and number of outbursts $\left(N_{\text {out }}\right)$ for dwarf novae studied. DN denotes dwarf nova. UG, ZC and SU denote U Gem, Z Cam and SU UMa-type systems, respectively. In the sixth column, the second number shows the number of superoutbursts for SU UMa-type systems. The sub-types and orbital periods are taken from Ritter \& Kolb (1998), Downes et al. (1997) and Lockley et al. (1999, for UY Pup).

\begin{tabular}{|c|c|c|c|c|c|}
\hline System & $P_{\text {orb }}$ & Sub-type & Interval & $N_{\text {obs }}$ & $N_{\text {out }}$ \\
\hline GK Per & 47.92 & DN & $1961-1997$ & 17758 & 11 \\
\hline BV Cen & 14.64 & $\mathrm{UG}$ & $1954-1997$ & 19445 & 29 \\
\hline V442 Cen & 11.04 & $\mathrm{UG}$ & $1954-1997$ & 17425 & 140 \\
\hline RU Peg & 8.99 & UG & $1961-1997$ & 34414 & 105 \\
\hline MU Cen & 8.21 & UG & $1954-1997$ & 9758 & 64 \\
\hline TT Crt & 7.30 & UG & $1989-1997$ & 2049 & 2 \\
\hline SS Cyg & 6.60 & UG & $1896-1992$ & 29388 & 683 \\
\hline BV Pup & 5.4 & UG & $1959-1997$ & 5203 & 7 \\
\hline CZ Ori & 5.15 & UG & $1954-1997$ & 6719 & 62 \\
\hline TW Vir & 4.38 & UG & $1955-1997$ & 7872 & 62 \\
\hline SS Aur & 4.39 & UG & $1961-1997$ & 24126 & 182 \\
\hline U Gem & 4.25 & UG & $1963-1995$ & 25707 & 79 \\
\hline UZ Ser & 4.15 & $\mathrm{UG}$ & $1955-1997$ & 6164 & 27 \\
\hline UU Aql & 3.37 & $\mathrm{UG}$ & $1984-1997$ & 4349 & 29 \\
\hline AT Ara & & $\mathrm{UG}$ & $1958-1997$ & 6778 & 23 \\
\hline FQ Sco & & $\mathrm{UG}$ & $1954-1997$ & 5827 & 8 \\
\hline $\mathrm{Z}$ Cam & 6.96 & $\mathrm{ZC}$ & $1963-1995$ & 39945 & 266 \\
\hline AH Her & 6.19 & $\mathrm{ZC}$ & 1963-1995 & 21586 & 335 \\
\hline AT Cnc & 5.73 & $\mathrm{ZC}$ & $1975-1997$ & 3485 & 46 \\
\hline $\mathrm{HL} \mathrm{CMa}$ & 5.15 & $\mathrm{ZC}$ & $1981-1997$ & 8906 & 115 \\
\hline RX And & 5.04 & $\mathrm{ZC}$ & $1963-1995$ & 37170 & 394 \\
\hline WW Cet & 4.24 & $\mathrm{ZC}$ & $1968-1997$ & 5745 & 45 \\
\hline CN Ori & 3.92 & $\mathrm{ZC}$ & $1961-1997$ & 12368 & 257 \\
\hline KT Per & 3.91 & $\mathrm{ZC}$ & $1967-1997$ & 14666 & 266 \\
\hline UY Pup & 10.22 & $\mathrm{ZC}$ & $1979-1997$ & 3536 & 13 \\
\hline TU Men & 2.82 & $\mathrm{SU}$ & $1963-1997$ & 15956 & $14-26$ \\
\hline BR Lup & 1.90 & $\mathrm{SU}$ & $1987-1997$ & 2221 & $4-0$ \\
\hline CU Vel & 1.86 & $\mathrm{SU}$ & $1954-1997$ & 20297 & $20-27$ \\
\hline WX Hyi & 1.80 & $\mathrm{SU}$ & $1971-1997$ & 21425 & $88-42$ \\
\hline $\mathrm{Z}$ Cha & 1.79 & $\mathrm{SU}$ & $1954-1997$ & 31431 & $28-47$ \\
\hline VW Hyi & 1.78 & $\mathrm{SU}$ & $1953-1997$ & 51234 & $445-86$ \\
\hline EK $\operatorname{Tr} A$ & 1.53 & $\mathrm{SU}$ & $1954-1997$ & 14681 & $8-18$ \\
\hline OY Car & 1.51 & $\mathrm{SU}$ & $1963-1997$ & 19928 & $12-15$ \\
\hline V436 Cen & 1.50 & $\mathrm{SU}$ & $1958-1997$ & 14278 & $6-16$ \\
\hline AQ Eri & 1.46 & $\mathrm{SU}$ & $1976-1997$ & 8041 & $1-0$ \\
\hline SW UMa & 1.36 & $\mathrm{SU}$ & $1961-1997$ & 7868 & $6-12$ \\
\hline
\end{tabular}

systems, mean values of the parameters are near the means reported in SM84.

\section{Results and discussion}

For correlation analysis, we combined our data sample with the data in SM84. Assuming a linear relation, we evaluated the correlations between the mean parameters and the intrinsic parameters. Table 3 lists the correlation coefficients $(r)$. The cycle time can give spurious correlations, since this parameter includes the outburst duration and quiescent interval. It may be more meaningful to explore the correlations between the parameters and the adjacent quiescent intervals (Cannizzo \& Mattei 1992). Hence, we did not use the mean cycle time in the analysis except for the $P_{\text {orb }}: C$ correlation.

\subsection{Correlations with the intrinsic parameters}

The white dwarf mass $M_{1}$ appears correlated with the outburst duration $W$ and decline rate $\tau_{\mathrm{d}}$. These correlations are much weaker in previous studies (SM84, Gieger 1987). Because the white dwarf mass has a moderate effect on the long-term light curve parameters, $M_{1}: W$ and $M_{1}: \tau_{\mathrm{d}}$ 
Table 2. The mean values of outburst durations $W$ (days), quiescent intervals $Q$ (days), cycle times $C$ (days), rise rates $\tau_{\mathrm{r}}$ and decline rates $\tau_{\mathrm{d}}$ (days $/ \mathrm{mag}$ ) for dwarf novae studied. $L_{2}$ and $L_{3}$ (magnitudes) are the second and third reference levels, respectively. Normal denotes the normal outbursts, super the superoutbursts.

\begin{tabular}{|c|c|c|c|c|c|c|c|}
\hline System & $W$ & $Q$ & $C$ & $\tau_{\mathrm{r}}$ & $\tau_{\mathrm{d}}$ & $L_{2}$ & $L_{3}$ \\
\hline \multicolumn{7}{|l|}{ normal } & 11.0 \\
\hline $\begin{array}{l}\text { BV Cen } \\
\text { V } 442 \text { Cen }\end{array}$ & $\begin{array}{c}25.1 \\
6.6\end{array}$ & $\begin{array}{c}159.4 \\
25.8\end{array}$ & $\begin{array}{c}166.6 \\
32.0\end{array}$ & $\begin{array}{l}6.7 \\
0.9\end{array}$ & $\begin{array}{l}5.7 \\
2.4\end{array}$ & $\begin{array}{l}12.8 \\
13.2\end{array}$ & $\begin{array}{l}11.5 \\
12.0\end{array}$ \\
\hline RU Peg & 16.0 & 60.4 & 76.4 & 1.3 & 3.1 & 12.5 & 11.0 \\
\hline MU Cen & 11.3 & 32.3 & 44.9 & 1.4 & 3.1 & 14.0 & 12.5 \\
\hline TT Crt & & & & & 2.5 & 13.8 & 13.1 \\
\hline SS Cyg & 19.6 & 31.0 & 50.2 & 1.0 & 2.7 & 11.0 & 9.0 \\
\hline BV Pup & 8.8 & & 30.2 & 0.9 & 3.8 & 14.0 & 12.5 \\
\hline CZ Ori & 9.1 & 16.0 & 26.4 & 1.1 & 2.7 & 13.5 & 12.5 \\
\hline TW Vir & 6.0 & 22.0 & 28.1 & 0.4 & 0.9 & 13.5 & 12.2 \\
\hline SS Aur & 12.2 & 40.8 & 51.4 & 0.6 & 1.7 & 14.0 & 11.5 \\
\hline $\mathrm{U}$ Gem & 17.8 & 105.9 & 132.3 & 0.4 & 1.3 & 13.0 & 10.0 \\
\hline UZ Ser & 7.6 & 16.1 & 24.7 & 1.4 & 2.2 & 13.5 & 12.8 \\
\hline UU Aql & 9.2 & 34.5 & 47.7 & 0.4 & 1.0 & 14.0 & 12.0 \\
\hline AT Ara & 11.6 & 79.1 & 96.8 & 1.3 & 3.3 & 13.4 & 12.8 \\
\hline FQ Sco & 7.0 & 55.8 & 61.0 & 1.0 & 2.4 & 13.3 & 12.4 \\
\hline UG mean & 12.0 & 52.2 & 62.0 & 1.3 & 2.6 & & \\
\hline Z Cam & 12.9 & 12.0 & 24.6 & 0.8 & 2.9 & 12.5 & 11.0 \\
\hline AH Her & 15.1 & 5.0 & 19.7 & 1.6 & 2.0 & 13.5 & 12.0 \\
\hline AT Cnc & 8.0 & 8.4 & 15.9 & 0.7 & 2.0 & 14.0 & 13.0 \\
\hline $\mathrm{HL} \mathrm{CMa}$ & 10.8 & 5.7 & 17.0 & 0.6 & 1.4 & 13.5 & 12.0 \\
\hline RX And & 8.6 & 8.4 & 16.6 & 0.6 & 1.3 & 13.0 & 12.0 \\
\hline WW Cet & 9.0 & 35.9 & 45.2 & 0.6 & 1.3 & 13.5 & 11.5 \\
\hline CN Ori & 13.0 & 5.1 & 18.2 & 1.7 & 2.7 & 13.8 & 12.5 \\
\hline KT Per & 8.2 & 11.5 & 19.5 & 0.6 & 1.5 & 14.0 & 12.5 \\
\hline UY Pup & 15.4 & 82.2 & 84.6 & 0.9 & 4.2 & 14.0 & 13.5 \\
\hline $\mathrm{ZC}$ mean & 11.2 & 19.4 & 29.0 & 0.9 & 2.1 & & \\
\hline TU Men & 2.3 & 1.9 & 4.3 & 0.3 & 0.7 & 13.8 & 13.0 \\
\hline BR Lup & 3.8 & 21.5 & 25.9 & 0.4 & 1.1 & 14.6 & 13.7 \\
\hline CU Vel & 4.5 & 69.4 & 74.6 & 0.5 & 1.1 & 13.3 & 12.5 \\
\hline WX Hyi & 2.9 & 8.7 & 11.2 & 0.5 & 0.9 & 13.9 & 13.0 \\
\hline Z Cha & 3.7 & 13.4 & 17.2 & 0.5 & 1.0 & 13.8 & 12.9 \\
\hline VW Hyi & 4.0 & 23.5 & 27.8 & 0.3 & 0.7 & 12.5 & 10.0 \\
\hline EK $\operatorname{Tr} A$ & 3.0 & 164.2 & 167.2 & 0.5 & 0.7 & 13.4 & 12.9 \\
\hline OY Car & 4.7 & 9.4 & 13.5 & 0.4 & 0.8 & 14.0 & 13.0 \\
\hline V436 Cen & 3.3 & 80.8 & 85.4 & 0.4 & 0.6 & 14.0 & 12.9 \\
\hline AQ Eri & 3.4 & & & 0.7 & 1.2 & 13.7 & 12.8 \\
\hline SW UMa & 7.6 & 23.8 & 29.5 & 0.1 & 0.6 & 14.5 & 12.8 \\
\hline SU mean & 4.3 & 41.7 & 45.7 & 0.4 & 0.8 & & \\
\hline \multicolumn{8}{|l|}{ super } \\
\hline TU Men & 9.2 & 187.4 & 202.1 & 0.4 & 1.8 & 13.8 & 12.2 \\
\hline CU Vel & 16.3 & 387.5 & 393.3 & 0.5 & 2.0 & 13.3 & 11.4 \\
\hline WX Hyi & 13.9 & 165.2 & 179.7 & 0.6 & 1.7 & 13.9 & 12.5 \\
\hline Z Cha & 14.0 & 230.5 & 228.7 & 0.5 & 3.2 & 13.8 & 12.5 \\
\hline VW Hyi & 16.2 & 167.7 & 182.5 & 0.3 & 0.9 & 12.5 & 10.0 \\
\hline EK $\operatorname{Tr} A$ & 13.0 & 519.4 & 530.9 & 0.5 & 1.4 & 13.4 & 12.0 \\
\hline OY Car & 18.1 & 280.9 & 325.1 & 0.6 & 1.4 & 14.0 & 12.5 \\
\hline V436 Cen & 15.3 & 393.5 & 403.8 & 0.3 & 1.1 & 14.0 & 12.5 \\
\hline SW UMa & 23.1 & 324.9 & 352.8 & 0.3 & 0.9 & 15.0 & 11.0 \\
\hline mean & 15.5 & 295.2 & 311.0 & 0.4 & 1.6 & & \\
\hline
\end{tabular}

correlations can be expected from the disk instability model (Cannizzo et al. 1988).

Correlations of the secondary mass $M_{2}$ with the outburst duration, rise rate and decline rate are consequences of the orbital period correlations with these quantities, since the secondary star is governed by the orbital period. Correlations of the orbital period with the long-term light curve parameters are important for a correct understanding of the outburst models. The data set gives the following relationships:

$$
\begin{aligned}
& W(\text { days })=1.56( \pm 0.16) P_{\text {orb }}(\text { hrs })+1.87( \pm 0.81) \\
& \tau_{\mathrm{r}}(\text { days } / \mathrm{mag})=0.12( \pm 0.02) P_{\text {orb }}(\text { hrs })+0.26( \pm 0.11) \\
& \tau_{\text {d }}(\text { days } / \mathrm{mag})=0.33( \pm 0.03) P_{\text {orb }}(\text { hrs })+0.27( \pm 0.16)
\end{aligned}
$$

Table 3. Linear correlation coefficients for mean parameters of normal outburtsts. $M_{1}$ and $M_{2}$ denote the white dwarf and the secondary masses, respectively. The number in parenthesis is number of the systems in correlation. Physical-system parameters are taken from Ritter \& Kolb (1998).

\begin{tabular}{lcccc}
\hline \hline & $W$ & $Q$ & $\tau_{\mathrm{r}}$ & $\tau_{\mathrm{d}}$ \\
\hline$P_{\text {orb }}$ & $0.85(40)$ & $0.28(39)$ & $0.65(40)$ & $0.87(42)$ \\
$M_{1}$ & $0.70(22)$ & $0.34(19)$ & $0.19(23)$ & $0.49(23)$ \\
$M_{2}$ & $0.77(24)$ & $0.21(24)$ & $0.83(23)$ & $0.84(23)$ \\
$W$ & & $0.10(33)$ & $0.57(42)$ & $0.73(43)$ \\
$Q$ & & & $-0.08(40)$ & $0.34(41)$ \\
$\tau_{\mathrm{r}}$ & & & & $0.77(41)$ \\
\hline
\end{tabular}

GK Per is excluded from the relationships due to high scatter. We also exclude V442 Cen and BV Cen from

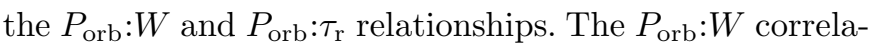
tion is plotted in Fig. 2. Orbital periods of AT Ara and FQ Sco are unknown (Ritter \& Kolb 1998). Relations of the orbital period may be used to predict the orbital periods for these systems. We can predict orbital periods of 6.2 and 3.3 hours for AT Ara and FQ Sco, respectively, from the $P_{\text {orb }}: W$ relationship.

Combining cycle times given in Richter \& Brauer (1989) with our data, we obtain a considerably larger data sample consisting of 57 systems. This data set does not give a $P_{\text {orb }}: C$ correlation $(r=0.24)$, although this correlation is expected from the disk instability model (Cannizzo et al. 1988).

\subsection{Other correlations}

The correlations were run for each individual system with more than 20 outbursts measured. The linear correlation coefficients are listed in Table 4 . In the following, we use the subscripts " $i-1$ " and " $i$ " to denote the parameters for intervals preceeding and following the " $i$ th" outburst, respectively.

A relation of the outburst duration with the preceeding quiescent interval is expected from the disk instability model. On the other hand, the mass-transfer burst model predicts a correlation of the outburst duration with the following quiescent interval (Gieger 1987). These predictions can be tested by using the $W_{i}: Q_{i-1}$ and $W_{i}: Q_{i}$ correlations. Table 4 shows that the systems in this study do not generally confirm either of these predictions.

For V442 Cen, SS Cyg and U Gem, wide and narrow outbursts tend to alternate: $W_{i}: W_{i+1}$. There are somewhat weaker (but still significant) $W_{i}: W_{i+1}$ correlations for $\mathrm{AH}$ Her, HL CMa and KT Per. A comparison of the correlations in Table 4 with the correlations in SM84 shows that we find different results for some systems. For example, we do not confirm $W_{i}: Q_{i-1}$ and $W_{i}$ : $Q_{i}$ correlations suggested in SM84 for RX And and AH Her, 
Table 4. Linear correlation coefficients for individual systems. Blanks mean $N<20$. Asterisks are used to show correlation coefficients $\geq 0.40$.

\begin{tabular}{lcccccccccc}
\hline \hline System & $W_{i}: W_{i+1}$ & $W_{i}: Q_{i}$ & $W_{i}: Q_{i-1}$ & $W_{i}: \tau_{\mathrm{r}_{\mathrm{i}}}$ & $W_{i}: \tau_{\mathrm{d}_{\mathrm{i}}}$ & $Q_{i}: \tau_{\mathrm{r}_{\mathrm{i}}}$ & $Q_{i}: \tau_{\mathrm{d}_{\mathrm{i}}}$ & $Q_{i-1}: \tau_{\mathrm{r}_{\mathrm{i}}}$ & $Q_{i-1}: \tau_{\mathrm{d}_{\mathrm{i}}}$ & $Q_{i}: Q_{i-1}$ \\
\hline V442 Cen & $-0.45^{*}$ & 0.29 & -0.03 & -0.17 & 0.06 & -0.34 & -0.04 & 0.02 & -0.29 \\
RU Peg & 0.04 & 0.31 & 0.06 & 0.14 & 0.08 & -0.14 & -0.14 & 0.00 & 0.09 \\
SS Cyg & $-0.47^{*}$ & 0.20 & 0.23 & 0.24 & 0.26 & -0.02 & -0.18 & -0.24 & -0.16 & $0.42^{*}$ \\
CZ Ori & & & & 0.19 & 0.02 & & & & \\
SS Aur & 0.06 & $0.41^{*}$ & $0.52^{*}$ & 0.30 & 0.27 & -0.07 & -0.11 & -0.22 & -0.18 & $0.40^{*}$ \\
U Gem & $-0.40^{*}$ & -0.09 & $0.49^{*}$ & 0.17 & 0.06 & 0.11 & 0.00 & -0.13 & -0.32 & \\
Z Cam & -0.20 & -0.01 & 0.24 & 0.04 & -0.06 & -0.14 & -0.09 & -0.19 & -0.10 & $0.45^{*}$ \\
AH Her & -0.39 & -0.09 & 0.16 & 0.22 & 0.12 & 0.25 & -0.04 & -0.25 & 0.07 & 0.16 \\
HL CMa & -0.31 & -0.16 & 0.35 & 0.26 & 0.38 & 0.02 & -0.02 & -0.02 & -0.09 & 0.31 \\
RX And & 0.06 & 0.16 & 0.28 & $0.41^{*}$ & 0.15 & 0.12 & -0.15 & 0.16 & -0.09 \\
CN Ori & -0.26 & -0.27 & -0.01 & 0.24 & 0.20 & -0.23 & -0.36 & $-0.56^{*}$ & -0.15 & $0.58^{*}$ \\
KT Per & -0.37 & 0.15 & 0.18 & 0.28 & 0.28 & 0.06 & 0.08 & -0.02 & 0.04 & $0.61^{*}$ \\
WX Hyi & $0.40^{*}$ & -0.23 & 0.03 & -0.05 & 0.01 & 0.07 & -0.13 & 0.33 & 0.32 & \\
VW Hyi & 0.02 & 0.22 & $0.45^{*}$ & 0.01 & 0.08 & -0.11 & -0.07 & -0.28 & 0.03 & 0.38 \\
\hline
\end{tabular}

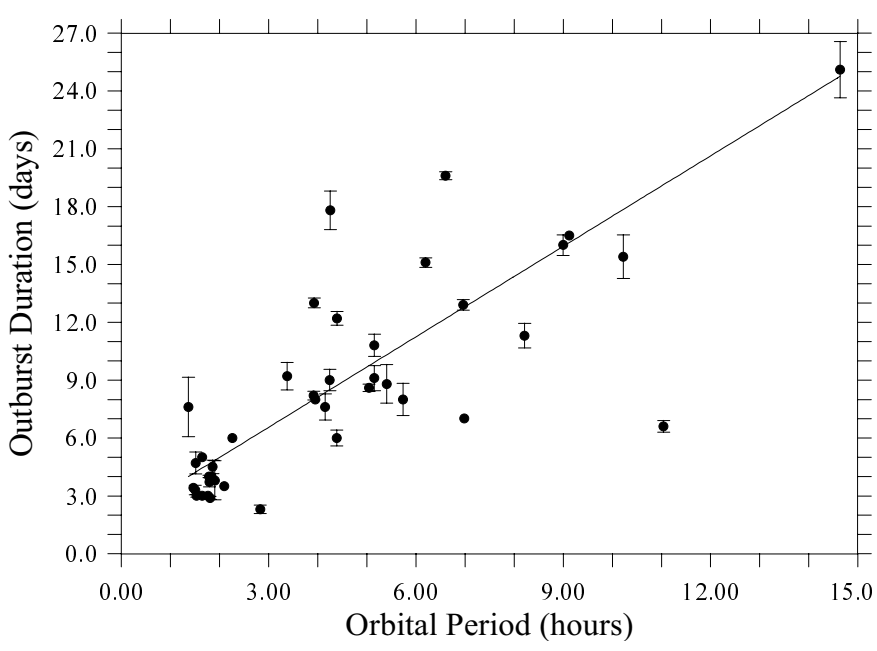

Fig. 2. The $P_{\text {orb }}: W$ relationship for normal outbursts of dwarf novae. Solid line shows the linear fit. Standard deviations of mean values are represented by bars.

respectively. The $Q_{i}: Q_{i-1}$ correlation implies that quiescent intervals of a given duration tend to occur together for about one third of the systems in Table 4. Our findings on SS Cyg, VW Hyi and Z Cam are in accord with the results given in Cannizzo \& Mattei (1992), Mohanty $\&$ Schlegel (1995) and Oppenheimer et al. (1998).

\subsection{Frequency distributions}

The separation into "wide" and "Narrow" outbursts for dwarf novae has been previously reported (e.g., van Paradijs 1983, SM84). This distinction of outburst durations is very clear for the SU UMa-type systems, since they possess normal (narrow) outbursts and super(wide)outbursts. The following U Gem and Z Camtype systems show obvious evidence of a bimodal distribution: AH Her, AT Ara, AT Cnc, CZ Ori, HL CMa, KT Per, RU Peg, RX And, SS Aur, SS Cyg, TW Vir, U Gem, UU Aql, UZ Ser, WW Cet and Z Cam. It is not clear whether GK Per, TT Crt, BV Pup, FQ Sco, and UY Pup have the bimodal nature of outburst duration. It is inter- esting to note that the distributions of outburst durations for CN Ori and MU Cen are not bimodal. BV Cen does not give an indication of a bimodal distribution, but it is not clear if this is due to sampling effects. Some examples of the frequency distributions can be seen in Fig. 3.

Cycle times and quiescent intervals exhibit asymmetrical distributions. The only exception is HL CMa. This system has a bimodal distribution of cycle times.

We select narrow and wide outbursts of the systems by applying bimodal gaussian fits to the frequency distributions. Correlations of the mean duration for narrow outbursts with the orbital period will be given in the next subsection. Here, it should be pointed out that the mean outburst duration is correlated with the orbital period for wide outbursts in Z Cam and U Gem-type systems, with $r=0.59$. Van Paradijs (1983) indicates that this correlation is absent. Due to high scatter, normal outbursts of SU UMa-type systems are not taken into account in this relation.

Smak (1999a) predicts that the decline rates should be higher in the case of wide outbursts. This effect is very clear for narrow and wide outbursts of SU UMa-type systems (see Table 1). For most of the Z Cam and U Gemtype systems, difference between the mean decline rates of narrow and wide outbursts is only $\sim 10 \%$ of the mean decline rate of narrow outbursts. This difference is $\sim 50 \%$ for HL CMa, TW Vir and UU Aql, and $\sim 30 \%$ for AT Ara and UZ Ser.

\subsection{The viscosity parameter $\alpha_{\text {hot }}$}

The outburst characteristics of dwarf novae are very sensitive to the viscous time-scale that is defined by the viscosity parameter $\alpha_{\text {hot }}$ on the hot branch of the $\Sigma-T_{\mathrm{e}}$ relation of the disk instability model (Meyer \& MeyerHofmeister 1981; Smak 1984; Cannizzo et al. 1988; Smak 1998, 1999a and 1999b). Theoretical correlations of the orbital period with the outburst time-scales provide an important opportunity for an observational determination of the viscosity parameter. Previous efforts in this regard using compilations from several sources have been made by 

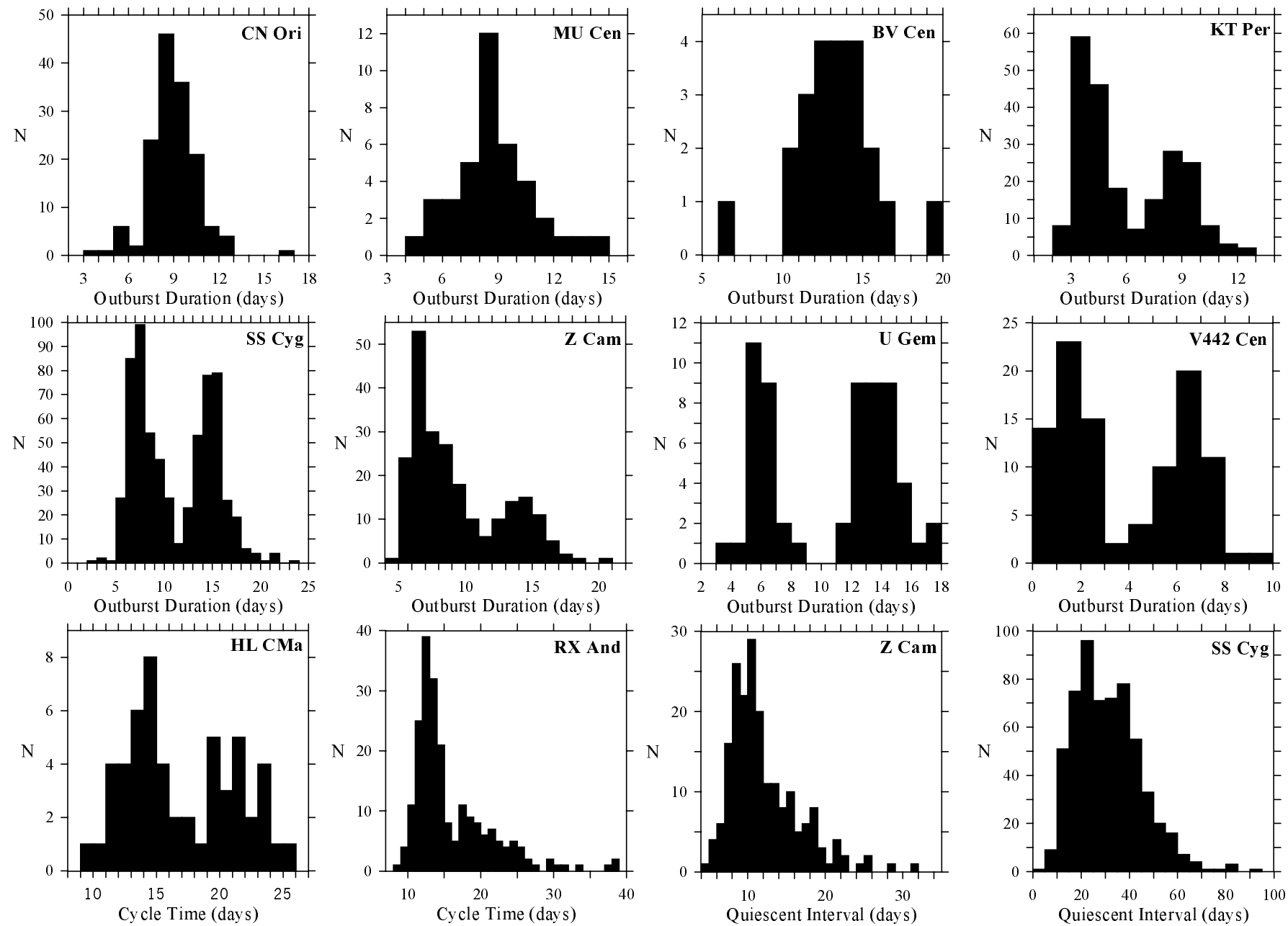

Fig. 3. Some examples from the frequency distributions of the long-term light curve parameters.

Gieger (1987), Cannizzo et al. (1988) and Smak (1999b, hereafter referred to as S99b). The observational data in S99b were taken from van Paradijs (1983).

Theoretical outburst durations and decline rates of narrow outbursts are listed in Smak (1998) for two different $\alpha_{\text {hot }}$ prescriptions. Since the outburst duration in Smak (1998) is defined at a reference level 1 mag below the outburst maximum, we recalculated the mean outburst durations ( $W_{1}$, reduced outburst duration) at this reference level and decline rates $\left(\tau_{\mathrm{d}_{\mathrm{n}}}\right)$ for narrow outbursts of the systems. We also recalculated the reduced durations of narrow outbursts for some systems in SM84 and van Paradijs (1983). We limit our data sample by the dwarf novae with $P_{\text {orb }}<10^{\mathrm{h}}$. Means for $W_{1}$ and $\tau_{\mathrm{d}_{\mathrm{n}}}$ are listed in Table 5. In the following, we compare the observational $P_{\mathrm{orb}}: W_{1}$ and $P_{\mathrm{orb}}: \tau_{\mathrm{d}_{\mathrm{n}}}$ relations with their theoretical counterparts. We use nearly the same notation as in S99b.

$P_{\text {orb }}: W_{1}$ relation: The relationship between $W_{1}$ and $P_{\text {orb }}$ (Fig. 4) can be expressed as either $W_{1}=C_{\mathrm{w}}^{\prime} P_{\text {orb }}^{\beta}$ or $W_{1}=C_{\mathrm{w}} P_{\text {orb }}$. For the non-linear case, we get $C_{\mathrm{w}}^{\prime}=$ $1.07 \pm 0.10$ and $\beta=0.88 \pm 0.09$ from the data set in Table 5 . Theoretical value of $\beta$ is 0.74 in Eq. (6) of S99b. In the linear case, we calculate $C_{\mathrm{w}}=0.88 \pm 0.07$. Reduced outburst

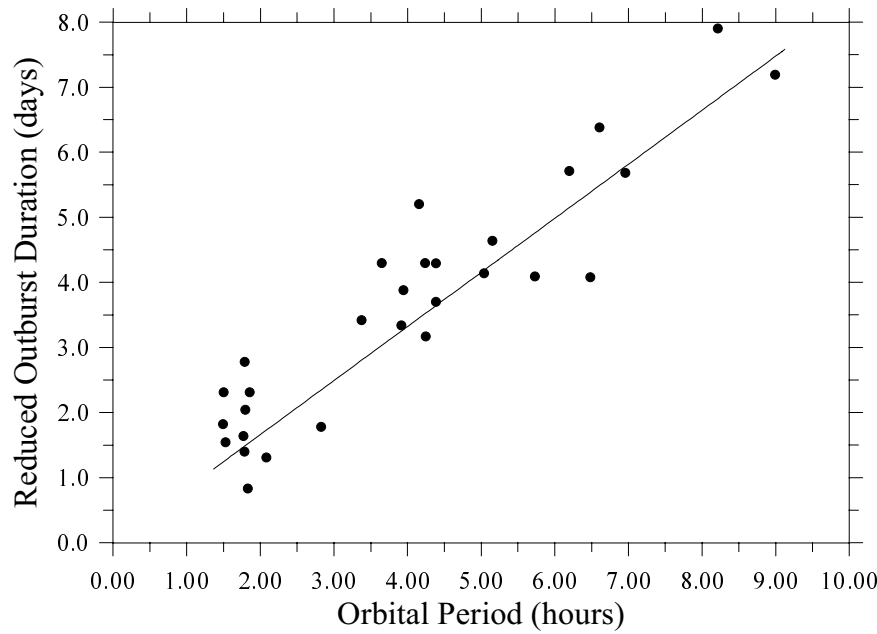

Fig. 4. The $P_{\mathrm{orb}}: W_{1}$ relation for narrow outbursts of dwarf novae. Solid line corresponds to the model data with $\alpha_{\text {hot }}=0.2$.

durations of CN Ori, HL CMa, OY Car and SW UMa are excluded from the $P_{\mathrm{orb}}: W_{1}$ relations due to high scatter. Since the dispersion in the linear case is lower, we can simply use the linear approximation. A linear fit to the theoretical outburst durations in Smak (1998) gives 
Table 5. The mean reduced durations $\left(W_{1}\right)$ and decline rates $\left(\tau_{\mathrm{d}}\right)$ of narrow outbursts. Orbital periods for AT Ara and FQ Sco are computed from Eq. (1) of this study. In fifth column, vP83 denotes van Paradijs (1983).

\begin{tabular}{|c|c|c|c|c|}
\hline System & $\begin{array}{c}P_{\text {orb }} \\
\text { (hours) }\end{array}$ & $\begin{array}{c}W_{1} \\
\text { (days) }\end{array}$ & $\begin{array}{c}\tau_{\mathrm{d}} \\
\text { (days/mag) }\end{array}$ & Reference \\
\hline SY Cnc & 9.12 & & 2.3 & SM84 \\
\hline RU Peg & 8.99 & 7.2 & 3.2 & this work \\
\hline MU Cen & 8.21 & 7.9 & 3.1 & this work \\
\hline EM Cyg & 6.98 & & 3.6 & SM84 \\
\hline Z Cam & 6.96 & 5.7 & 2.9 & this work \\
\hline SS Cyg & 6.60 & 6.4 & 2.5 & this work \\
\hline AT Ara & 6.48 & 4.1 & 2.3 & this work \\
\hline TZ Per & 6.25 & & 2.5 & SM84 \\
\hline AH Her & 6.19 & 5.7 & 1.8 & this work \\
\hline $\mathrm{AT} \mathrm{Cnc}$ & 5.73 & 4.1 & 1.8 & this work \\
\hline $\mathrm{CZ}$ Ori & 5.15 & 4.6 & 2.3 & this work \\
\hline $\mathrm{HL} \mathrm{CMa}$ & 5.15 & 7.0 & 1.1 & this work \\
\hline RX And & 5.04 & 4.1 & 1.3 & this work \\
\hline SS Aur & 4.39 & 4.3 & 1.6 & this work \\
\hline TW Vir & 4.38 & 3.7 & 0.8 & this work \\
\hline U Gem & 4.25 & 3.2 & 1.3 & this work \\
\hline WW Cet & 4.24 & 4.3 & 1.2 & this work \\
\hline UZ Ser & 4.15 & 5.2 & 1.8 & this work \\
\hline X Leo & 3.95 & 3.9 & 1.1 & vP83, SM84 \\
\hline $\mathrm{CN}$ Ori & 3.92 & 8.9 & 2.7 & this work \\
\hline KT Per & 3.91 & 3.3 & 1.4 & this work \\
\hline AR And & 3.91 & & 0.6 & SM84 \\
\hline AB Dra & 3.65 & 4.3 & 2.5 & vP83 \\
\hline UU Aql & 3.37 & 3.4 & 0.7 & this work \\
\hline TU Men & 2.82 & 1.8 & 0.7 & this work \\
\hline YZ Cnc & 2.08 & 1.3 & 0.9 & SM84 \\
\hline CU Vel & 1.86 & 2.3 & 1.1 & this work \\
\hline SU UMa & 1.83 & 0.8 & 1.4 & SM84 \\
\hline WX Hyi & 1.80 & 2.0 & 0.9 & this work \\
\hline $\mathrm{Z}$ Cha & 1.79 & 2.8 & 1.0 & this work \\
\hline VW Hyi & 1.78 & 1.4 & 0.7 & this work \\
\hline AY Lyr & 1.77 & 1.6 & 0.9 & vP83, SM84 \\
\hline IR Gem & 1.64 & & 0.8 & SM84 \\
\hline TY Psc & 1.64 & & 0.7 & SM84 \\
\hline $\mathrm{EK} \operatorname{Tr} \mathrm{A}$ & 1.53 & 1.5 & 0.7 & this work \\
\hline OY Car & 1.51 & 4.0 & 0.8 & this work \\
\hline V436 Cen & 1.50 & 2.3 & 0.6 & this work \\
\hline UV Per & 1.49 & 1.8 & 1.2 & vP83 \\
\hline SW UMa & 1.36 & 6.0 & 0.6 & this work \\
\hline
\end{tabular}

$C_{\mathrm{w}}(0.1)=1.44 \pm 0.10$ and $C_{\mathrm{w}}(0.2)=0.83 \pm 0.05$ for $\alpha_{\text {hot }}=0.1$ and $\alpha_{\text {hot }}=0.2$, respectively. Comparing theoretical and observational fit parameters, we find $\alpha_{\text {hot }}=$ $0.19 \pm 0.01$ which is in agreement with the viscosity parameters given in S99b and Gieger (1987).

$P_{\text {orb }}: \tau_{d_{\mathrm{n}}}$ relation: For the non-linear case $\left(\tau_{\mathrm{d}_{\mathrm{n}}}=\right.$ $\left.C_{\tau}^{\prime} P_{\text {orb }}^{\beta}\right)$, we find $C_{\tau}^{\prime}=0.49 \pm 0.05$ and $\beta=0.74 \pm 0.09$. Note that this value of $\beta$ is the same as that predicted in Eq. (6) of S99b. The observational fit parameter in the linear case $\left(\tau_{\mathrm{d}_{\mathrm{n}}}=C_{\tau} P_{\mathrm{orb}}\right)$ is $C_{\tau}=0.35 \pm 0.03$. CN Ori, AB Dra, UV Per, SU UMa, SY Cnc and EM Cyg are omitted due to high scatter. The model data in Smak (1998) give $C_{\tau}(0.1)=0.74 \pm 0.04$ and $C_{\tau}(0.2)=0.42 \pm 0.02$ for $\alpha_{\text {hot }}=0.1$ and $\alpha_{\text {hot }}=0.2$, respectively. So, we get $\alpha_{\text {hot }}=0.22 \pm 0.01$ from a comparison between theoretical and observational fit parameters.

The mean durations and decline rates of narrow outbursts are correlated as expected: $\tau_{\mathrm{d}_{\mathrm{n}}}=(0.38 \pm 0.04) W_{1}$ with $r=0.97$. Deviations $\delta W_{1}$ and $\delta \tau_{\mathrm{d}_{\mathrm{n}}}$ from the $P_{\mathrm{orb}}: W_{1}$ and $P_{\mathrm{orb}}: \tau_{\mathrm{d}_{\mathrm{n}}}$ relations have a relatively weak correlation $(r \approx 0.50)$, which implies that the observed scatter in $P_{\text {orb }}: W_{1}$ and $P_{\text {orb }}: \tau_{\mathrm{d}_{\mathrm{n}}}$ correlations is probably due to intrinsic scatter in $W_{1}$ and $\tau_{\mathrm{d}_{\mathrm{n}}}$. Possible sources of the scatter should be the white dwarf mass $M_{1}$ and the mass transfer rate $M_{\mathrm{tr}}$. By using available data, we find that there is no correlation of $M_{1}$ with $W_{1}$ and $\tau_{\mathrm{d}_{\mathrm{n}}}$. This result is in agreement with the very weak dependence of the outburst time-scales on $M_{1}$, as predicted in S99b. S99b points out that physical meaning of the mass transfer rate is not quite obvious. However, $M_{\mathrm{tr}}$ may be suggested as a possible source of the scatter mentioned above, since the mass transfer rate has a considerable scatter for a given orbital period (Patterson 1984).

\section{Conclusions}

We have analyzed the long-term visual light curve parameters of 36 dwarf novae. New relations of the orbital period with the mean light curve parameters are given. Correlation of the white dwarf mass with outburst duration is much stronger than the correlations found in previous studies. We confirm some, but not all, of the correlations suggested in SM84 for individual systems. Almost all of dwarf novae with enough data have a bimodal distribution of outburst duration, except for CN Ori and MU Cen.

Non-linear approximations of the orbital period relations for narrow outbursts confirm the dependence of the outburst characteristics on orbital period as predicted in the disk instability model. From a comparison of the observational data with the theoretical outburst time-scales, we find that the value of the viscosity parameter of the disk instability model is $\alpha_{\text {hot }} \approx 0.2$.

Acknowledgements. We acknowledge the hundreds of the AAVSO and RASNZ observers worldwide. Thanks are due to Dr. F. M. Bateson for his helps in obtaining the data. We also thank Dr. T. Ozisik for developing the computer program used for measurements. Finally, the helpful comments of the referee are acknowledged. Part of this work was supported by the Research Fund of the University of Istanbul, Project Numbers: O-1013/25052001 and B-637/17072000.

\section{References}

Antipova, L. I. 1987, Ap\&SS, 131, 453

Bailey, J. 1975, J. Brit. astr. Ass., 86, 30

Cannizzo, J. K., Shafter, A. W., \& Wheeler, J. C. 1988, ApJ, 333,227

Cannizzo, J. K., \& Mattei, J. A. 1992, ApJ, 401, 642

Cannizzo, J. K. 1993, ApJ, 419, 318

Downes, R. A., Webbink, R. F., \& Shara, M. H. 1997, PASP, 109,345

Gieger, A. 1987, Acta Astron., 37, 29

Hameury, J. M., Menou, K., Dubus, G., Lasota, J. P., \& Hure, J. M. 1998, MNRAS, 298, 1048

Jacchia, L. G. 1975, J.A.A.V.S.O., 4, 49

Lockley, J. J., Wood, J. H., Jones, D. H. P., \& Mineshige, S. 1999, Ap\&SS, 226, 453 
Mattei, J. A. 1990, Optical Properties of Cataclysmic Variable Stars, in Proc. of the NATO ASI on Active Close Binaries, Active Close Binaries, ed. C. Ibanoglu (Kluwer, Dordrecht), 611

Meyer, F., \& Meyer-Hofmeister, E. 1981, A\&A, 104, L10

Mohanty, P., \& Schlegel, E. M. 1995, ApJ, 449, 330

Oppenheimer, B. D., Kenyon, S. J., \& Mattei, J. A. 1998, AJ, 115,1175

Patterson, J. 1984, ApJS, 54, 443

Richter, G. A., \& Brauer, H. J. 1989, Astron. Nachr., 310, 413

Ritter, H., \& Kolb, U. 1998, A\&AS, 129, 83

Smak, J. 1984, Acta Astron., 34, 161
Smak, J. 1985, Acta Astron., 35, 357

Smak, J. 1998, Acta Astron., 48, 677

Smak, J. 1999a, Acta Astron., 49, 383

Smak, J. 1999b, Acta Astron., 49, 391 (S99b)

Sterne, T. E., \& Campbell, L. 1934, Ann. Harward College Obs., 90, 189

Szkody, P., \& Mattei, J. A. 1984, PASP, 96, 988 (SM84)

van Paradijs, J. 1983, A\&A, 125, L16

Vogt, N. 1995, in ed. A. Bianchini, M. Della Valle, \& M. Orio, Cataclysmic Variables (Kluwer Academic Publ., The Netherlands), 359 